\title{
TOTALLY ENDOSCOPIC RESECTION OF ATRIAL MYXOMA: EARLY RESULTS OF 26 PATIENTS IN E HOSPITAL
}

\author{
Nguyen Hoang $\mathrm{Nam}^{1+}$, Nguyen Cong Huu ${ }^{1+}$, Tran Thuy Nguyen ${ }^{1+*}$, Hoang Van Trung ${ }^{1}$, Do Duc \\ Thinh $^{1}$, Ngo Thanh Hung ${ }^{1}$, Pham Thanh Dat ${ }^{1}$, Le Ngoc Thanh ${ }^{1}$, Nguyen Huu Uoc ${ }^{2 *}$
}

\section{ABSTRACT}

Objective: Myxoma is the most common form of non-malignant tumor that arises from connective tissue. Totally endoscopic surgery without robotic assistance can resect the entire atrial myxoma. This study aim to evaluate the early results of this surgical method.

Methods: From January 2019 to April 2021, 26 patients (20 females, 6 males, mean age $49.5 \pm 14.3$ ) were diagnosed with atrial myxoma. All tumors of those patients were resected by totally endoscopic surgery. We evaluated the early outcome of this method based on the following criteria: mortality rates, conversion to open surgery, cardiopulmonary bypass time, aortic cross-clamp time, postoperative time.

Results: Totally endoscopic surgery to resect atrial myxoma was successfully performed in all patients with surgical ports on the thoracic wall. The largest incision was not more than 1.5 $\mathrm{cm}$ in diameter. Mean cardiopulmonary bypass time was $134 \pm 39$ minutes, aortic cross-clamp time was $81.4 \pm 26.4$ minutes, mechanical ventilation time was $10.5 \pm 4.6$ hours, ICU length of stay was $2.1 \pm 0.9$ days, postoperative time was $6.9 \pm 5.4$ days. We had one case in which the excision of myxoma was performed contemporaneously with mitral valve annuloplasty.

Conclusions: Initial outcomes of totally endoscopic surgery to resect atrial myxomas were satisfactory. However, to fully evaluate the effectiveness of this method, we need to conduct a long-term follow-up of these patients.

Keywords: Myxoma, Minimally Invasive Surgery, Totally endoscopic surgery

\section{NTRODUCTION}

Primary cardiac tumors are uncommon, and the prevalence in the population is estimated between $0.0017 \%$ and $0.19 \%$. Most of primary tumors are benign (75\%), in which myxoma accounts for approximately $50 \%$ of cases. Cardiac myxoma, which is mainly developed in the atrium, is located in the left atrium in approximately $70 \%$ of cases, the right atrium for more than $15 \%$ and the ventricle or both atria for the rest. The atrial myxoma can lead to complications related to vascular occlusion, neurological injury, hemodynamic dysfunction caused by obstruction, pulmonary embolism, and pulmonary edema. Resection surgery should be performed at once the diagnosis was confirmed [1]. Minimally invasive or totally endoscopic surgery has been projected to be implemented increasingly in cardiovascular surgery. This method resulted in numerous advantages such as improved cosmetic features, less surgical trauma

1 Departement of Cardiothoracic Surgery- Cardiovascular CenterE Hospital

2 Cardiovascular and Thoracic Center- Viet Duc Hospital

+These authors contributed equally to this work

*Corresponding authors: Nguyen Huu Uoc, Nguyen Tran Thuy

Email: uocdhyhn101@yahoo.com.vn; drtranthuyvd@gmail.com

Address: 40 Trang Thi street - Ha Noi - Viet Nam

Received: 31 May 2021

Accepted: 02 July 2021 
and shorter postoperative period [2]. As reported by effective and satisfactory results of minimally invasive surgery in the treatment for mitral valve diseases, atrial septal defect and partial atrioventricular septal defect [3],[4], we performed totally endoscopic surgery for atrial myxoma resection with small skin incisions (the largest incision is $1.5 \mathrm{~cm}$ ) for operating surgical instruments. This report is aimed to evaluate the early results of this method.

\section{METHODS}

A total of 26 patients (20 females) underwent $3 \mathrm{D}$ totally thoracoscopic surgery to resect atrial myxomas from January 2019 to April 2021. All patients were diagnosed with atrial myxomas (19 left atrial myxomas, 7 right atrial myxomas) based on transthoracic echocardiography. A thoracic, abdominal and cranial computed tomography was carried out for general examination. Especially, computed tomography was used to determine tumor sites, and to screen whole-body for primary tumors originated from other organs, and to detect complications caused by pulmonary embolism. Besides, the patients should be examined carefully for vascular diseases as well as a history of previous thoracic surgery. Before being discharged, the patients who had atrial myxomas confirmed by pathological results were reexamined by echocardiography. This report was performed retrospectively by inspecting medical records and collected data in relation to operating time, cardiopulmonary bypass time, the amount of blood transfusion, mechanical ventilation time, recovery time, and postoperative time.

During the procedure, the patients were positioned supine on the right hemi-thorax elevated at 30 degrees with arms stretched along the body underwent general anesthesia using double lumen endotracheal tube. In addition, transesophageal echocardiography had been already established. Peripheral extracorporeal circulation was initiated by an arteriovenous configuration cannulated via the right femoral artery, femoral and internal jugular vein. All procedures for establishing peripheral extracorporeal circulation were performed with a Seldinger procedure using guide wire, which was monitored by transesophageal echocardiography. The trocarts of size $5 \mathrm{~mm}, 10 \mathrm{~mm}, 12 \mathrm{~mm}$ were placed in the $3^{\text {rd }}$ intercostal space in anterior axillary line, $4^{\text {th }}$ intercostal space in middle axillary line and $5^{\text {th }}$ intercostal space between anterior axillary line and midclavicular line on the right thorax, respectively. Cardiopulmonary bypass was implemented while only left lung was ventilated. After opening pericardium, the superior vena cava snare was inserted via endoscopic visualization. Insuffating carbon dioxide into the surgical field, afterward the atrial opening incision was depended on the location of the tumor confirmed by preoperative echocardiography. Most of myxoma tumors are located in the left atrium and adhered to the atrial septum, thus the incision of the right atrium was parallel to the atrioventricular groove, and openning the left atrium via the oval fossa (if the atrial myxoma is attached to the left atrial wall, it can be opened directly into the left atrium). Resected atrial myxomas was put into a collecting endoscopic bag. Removing the myxomas via the $12 \mathrm{~mm}$ trocar was followed closure of the cardiac chambers. Different strategies for myocardial protection consisted of: resection of left atrial myxoma using cardioplegia via the aortic root with transthoracic Chidwood clamp, whereas resection of right atrial myxoma performed without aortic cross-clamp during beating heart surgery. Trocar $10 \mathrm{~mm}$ was used for the $3 \mathrm{D}$ endoscope. Surgical instruments, the snare of the 
superior vena cava was delivered via a $5 \mathrm{~mm}$ trocar. The manipulations of the endoscopic scissors, the needle holder, cardioplegia needle and myxoma excision are performed via the $12 \mathrm{~mm}$ trocar. Observing transesophageal echocardiography and controlling intracardiac air in the heart chambers. Pericardial and pleural drainage were inserted via the sites of trocar.

\section{Statistical Analysis}

Data were expressed as mean $\pm \mathrm{SD}$ for quantitative variables and number and percentage for qualitative variables. Data were inputted and analyzed with SPSS version 16.0.

\section{RESULTS}

A total of 26 patients with an average age of $49,5 \pm 14,3$ had clinical characteristics with variety symptoms of cardiac obstruction and embolism (Table 1). Dyspnea on exertion and chest pain were the most common symptoms. Most of tumors were located in the left atrium and arose in the region of the atrial septum (Table 2). Completely resection of tumors, no hospital death, and no converion to sternotomy were observed. The intraoperative and postoperative characteristics are shown in (Table 3). Femoral artery thrombectomy caused by peripheral embolism was performed contemporaneously with myxoma resection in one patient, and another patient underwent myxoma resection and mitral valve annuloplasty in a combination. There were no complications related to air embolism, reoperation for bleeding or hematoma in the pleural cavity.

\section{Table 1: Preoperative clinical symtoms}

\begin{tabular}{lc}
\hline Symtoms & No. of patients (\%) \\
\hline Cardiac symptoms & $14(53,8 \%)$ \\
- Dyspnea & $11(42,3 \%)$ \\
- Palpitation & $3(11,5 \%)$ \\
- Congestive heart failure & \\
Embolic symptoms & $4(15,4 \%)$ \\
- Central nervous system & $1(3,8 \%)$ \\
- Peripheral embolism & \\
Constitutional symptoms & 0 \\
- Fever & 0 \\
- Weight loss & $3(11,5 \%)$ \\
- Fatigue & \\
\hline
\end{tabular}

Table 2: Preoperative echocardiographic characteristics of atrial myxoma.

\begin{tabular}{|c|c|c|c|}
\hline \multicolumn{2}{|c|}{ Characteristics } & Right atrial myxoma & Left atrial myxoma \\
\hline \multicolumn{2}{|c|}{ No. of patients } & $7(26,9 \%)$ & $19(73,1 \%)$ \\
\hline \multirow{2}{*}{ Tumor site } & Atrial septum & $4(57,1 \%)$ & $18(94,7 \%)$ \\
\hline & Other sites & $3(42,9 \%)$ & $1(5,3 \%)$ \\
\hline \multicolumn{2}{|c|}{ Size $(\overline{\bar{X}} \pm s x) \mathrm{mm}$} & $45,4 \pm 18,9$ & $41,9 \pm 14,9$ \\
\hline
\end{tabular}


Table 3: Intraoperative and postoperative characteristics

\begin{tabular}{|l|l|}
\hline Characteristics & $\bar{X} \pm s x$ \\
\hline Cardiopulmonary bypass time (minutes) & $134 \pm 39$ \\
\hline Aortic crossclamp time ( minutes) & $81,4 \pm 26,4$ \\
\hline Mechanical ventilation time (h) & $10,5 \pm 4,6$ \\
\hline ICU length of stay (days) & $2,1 \pm 0,9$ \\
\hline Postoperative period (days) & $6,9 \pm 5,4$ \\
\hline
\end{tabular}

\section{DISCUSSION}

Since the first atrial myxoma case was diagnosed and successfully resected [5], surgical resection of atrial myxoma has been performed by conventional median sternotomy with extracorporeal circulation. Currently, with advances in science and technology, the improvement of surgical instruments, cardiopulmonary bypass technique have facilitated operations to be made via smaller incisons, minimally invasive or totally endoscopic surgery using robots. Numerous studies have shown the benefits of minimally invasive surgery in the treatment of mitral valve diseases [6], atrial septal defect [7]. Compared with conventional surgery, it has been found to have excellent results in cosmetic features, less pain, fewer blood transfusions and shorter recovery time. There has been several published studies on the success in managing atrial myxomas with minimally invasive surgery. According to Andres M. Pineda's study, 7 different reports, which were collected to compare between minimally invasive surgery (via right thoracotomy, partial sternotomy, endoscopic assistance) and total sternotomy, revealed that the patients who were operated by minimally invasive thoracotomy had a reduced of length of stay in intensive care unit and recovery time with the constant mortality rate [8].

A new advance in the minimally invasive surgery that reported by Falk and Changquing Gao is successful implementation of voicecontrolled robotic arm and complete De Vinci robot usage [9],[10]. However, totally endoscopic surgery can also be performed without robotic assistance as Shiqiang Yu's report which included 12 patients with atrial myxoma resected by totally thoracoscopic operation without robotic assistance achieved good results [11]. Highlights of total endoscopy are composed of the extremely cosmetic outcomes with smaller surgical scars caused by trocars, preserve integrity of sternum, reduced damage to thoracic muscles, less postoperative pain and superior visualisation of the cardiac structures. Depending on our hospital situation, we performed totally endoscopic surgery without robotic assistance to resect atrial myxoma.

Myxoma was completely resected with 3 surgical ports (right atrial myxoma) or 4 surgical ports (left atrial myxoma). In comparison with procedure performed by DA Murphi with totally robotic endoscopy, Changing Gao used 5 surgical ports comprised of 4 surgical ports for 1 $\mathrm{cm}$ trocar and 1 port for $1.5 \mathrm{~cm}$ trocar. In our study, the number of scars were less and the 
chest wall was slightly damaged [10], [12]. The mean cardiopulmonary bypass time was $134 \pm$ 39 minutes, the mean aortic cross-clamp time was $81,4 \pm 26,4$ minutes. They were longer than the time in Changing Gao's report $(98 \pm 20,5$ minutes, $45 \pm 11,9$ minutes) and Douglas A.Murphy's (103 \pm 40 minutes, $64 \pm 2$ minutes), respectively [12]. There's a number of reasons for this difference. Firstly, we didn't place a suction cannula in the left atrium, instead of intermittent suction via the main surgical port, thus the manipulation was interrupted causing the delay. Secondly, the patients with right atrial myxoma were operated by beating heart surgery without aortic clamping, which found difficult to

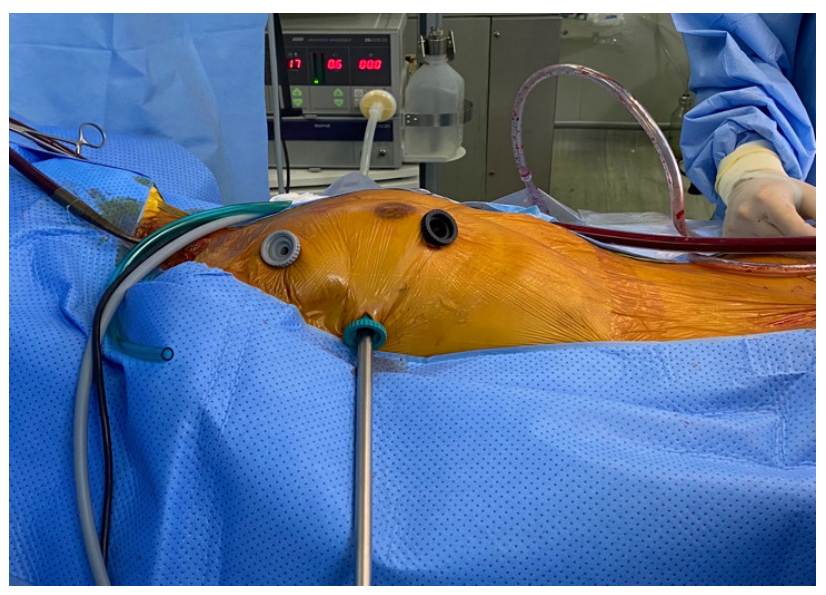

Figure 1: Set-up of surgical instruments

All patients underwent echocardiographic examination before discharge, and tumors was completely resected. However, this study only reported the success in a few clinical cases, hence we remark several following points: In resection of right atrial myxoma, during cardiopulmonary bypass was established, the superior and inferior vena cava were cannulated by using a guide wire, which could result in disrupting and dispersing the tumor. As a consequence, we recommend implementing transesophageal echocardiography resect the myxoma. Lastly, according to the two reports, the tumor was merely resected, by contrast, we had two patients required to repair the atrial septum and the mitral valve, respectively.

The length of stay in intensive care unit was $2,1 \pm 0,9$ days, the postoperative time was $6,9 \pm$ 5,4 days, which were the same as Changing Gao and Shiqiang Yu's reports. It revealed that the patients recovered quickly and was discharged from the hospital quite soon. There were no differences in surgical outcomes compared with the previously reports. There were no complications related to neurological event, embolism and re-exploration for bleeding.

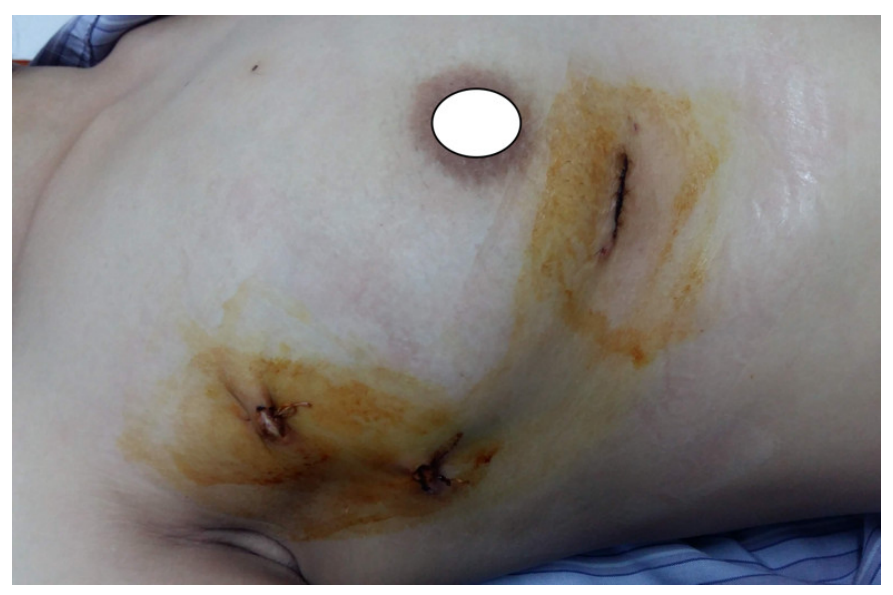

Figure 2: Postoperative scars

proactively in order to follow guide wire and venous cannulas, which restraints the impact on tumors. The risk of air embolism during opening right atrium requires combining techniques: lower head, carbon dioxide insufflation. The right atrium is only opened after filling carbon dioxide in the whole thoracic cavity insufflated continuously during tumor resection and closure of the right atrium. After the tumor was excised, it should be put into the collecting bag to prevent the risk of tumor dispersion. 


\section{CONCLUSION}

Initial outcomes of totally endoscopic surgery to resect atrial myxomas were satisfactory. However, to fully evaluate the effectiveness of this method, we need to conduct a long-term follow-up of these patients.

\section{REFERENCE}

1. Reynen K. Cardiac myxomas. N Engl J Med. 1995; 333(24):1610-1617.

2. Modi P, Hassan A, Chitwood WR Jr. Minimally invasive mitral valve surgery: a systematic review and meta-analysis (2008). Eur J Cardiothorac Surg. 2008; 34:943-952

3. Nguyen Cong Huu, Nguyen Hoang Nam, Nguyen Trung Hieu et al. Surgery with thoracoscopic support to close the atrial septal defect under extracorporeal circulation, beating heart, without aortic cross-clamp . Vietnam journal of endolaparoscopic surgery. 2014; 3(3): 29-33

4. Casselman FP, Slyke SV, Dom H, Lambrechts DL, Vermeulen Y, Vanermen H. Endoscopic mitral valve repair: feasible, reproductive, and durable. $\mathrm{J}$ Thorac Cardiovasc Surg. 2003; 125:273-282

5. Chitwood WR Jr. Clarence Crafoord and the first successful resection of a cardiac myxoma. Ann Thorac Surg. 1992 Nov;54(5):997-8.
6. Schmitto JD, Mokashi SA, Cohn LH. Minimally-Invasive Valve Surgery. Journal of the American College of Cardiology. 2010; 56(6):455-462

7. Argenziano $\mathrm{M}, \mathrm{Oz} \mathrm{MC}$, Kohmoto $\mathrm{T}$ et al. Totally endoscopic atrial septal defect repair with robotic assistance. Circulation. 2003;108 Suppl 1: II191-II194

8. Pineda AM, Santana O, Cortes-Bergoderi M, Lamelas J. Is a minimally invasive approach for resection of benign cardiac masses superior to standard full sternotomy? Interact Cardiovasc Thorac Surg. 2013;16(6):875-879.

9. Falk V, Walther $\mathrm{T}$, Autschbach $\mathrm{R}$ et al. Robot-assisted minimally invasive solo mitral valve operation. J Thorac Cardiovasc Surg. 1998 Feb;115(2):470-1.

10. Gao C, Yang M, Wang G et al. Excision of atrial myxoma using robotic technology. J Thorac Cardiovasc Surg. 2010 May;139(5):1282-1285.

11. Yu S, Xu X, Zhao B et al. Totally thoracoscopic surgical resection of cardiac myxoma in 12 patients. Ann Thorac Surg. 2010 Aug;90(2):674-6.

12. Murphy DA, Miller JS, Langford DA. Robotassisted endoscopic excision of left atrial myxomas. J Thorac Cardiovasc Surg. 2005 Aug;130(2):596-597 